\title{
Best-intentioned efforts aggravated AIDS pandemic
}

\section{The Origins of AIDS}

Jacques Pepin

Cambridge University Press; 2011.

$\mathbf{J}$ acques Pepin's remarkable book uses molecular biology, mathematics and social history to generate a sobering hypothesis for the origins of AIDS. Based on years of clinical experience in Africa and meticulous research, he lays the blame on iatrogenic causes: good intentions gone awry.

Iatrogenic theories of the origins of AIDS are not new. Pepin deals with a few. One rejected hypothesis was the idea that HIV lurked in polio vaccines tested in 1950s Africa. But the timing was wrong, the simian host was wrong, the location was wrong and the vaccines (when finally tested) did not contain HIV. Similarly he dispenses with the suggestion that AIDS originated in xenotransplantation of animal testicles promoted by Serge Voronoff. He also shows that early clusters of Kaposi's sarcoma (KS) cannot be construed as foci of unrecognized AIDS; KS arises from another virus.

Pepin's iatrogenic theory is different. He sets the stage carefully. A presentation of HIV types and chimpanzee types together with their geographic

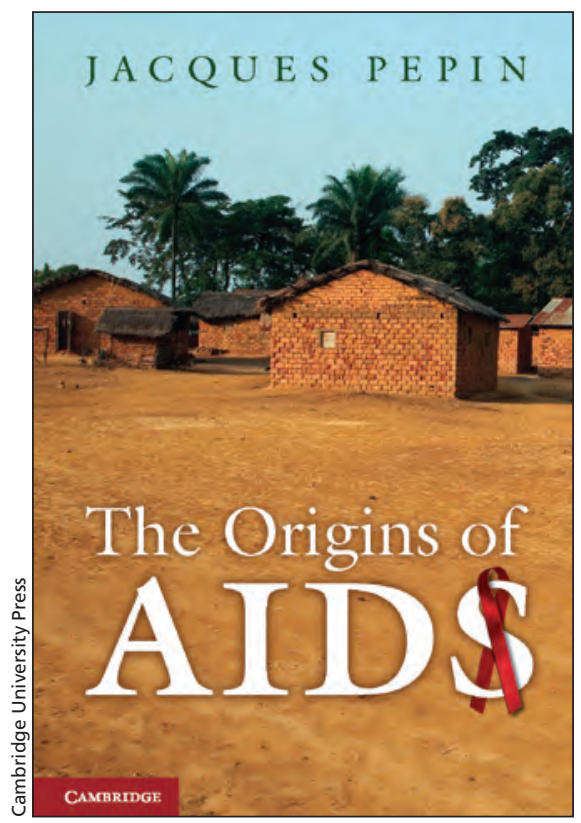

became infected from a chimpanzee. Interviews with indigenous people permit a realistic calculation of the risk of such injuries. Through genealogical analysis of molecular inheritance, the crucial injury is situated around 1921.

Next Pepin examines African sociopolitical history and the frightful conditions of colonialism: poverty, exploitation and disruption of long stable patterns of existence. Urban areas arose with many more men than women; forms of prostitution were

\section{Even the best-intentioned efforts aggravated, and possibly provoked, the pandemic of AIDS.}

distributions shows that only one chimpanzee harbors the SIV strain that became HIV-1. This exercise draws attention to Cameroon and the region of the Congo.

To explain the leap from apes to humans, Pepin subscribes to the "cut hunter" theory: a hunter, butcher or cook rampant. But Pepin argues, again through statistics, that the epidemic might not have been sustained sexually had the virus not been amplified earlier in other ways. Which is where his iatrogenic theory comes in.

This amplification, Pepin contends, occurred in medical campaigns for control of sleeping sickness, malaria, yaws, syphilis and other diseases. Detailed records held in European archives reveal that earnest health workers performed thousands of injections in remote villages and that their syringes and needles were inadequately sterilized. Using rates of seroconversion, established more recently in recipients of infected blood and those with needle-stick injuries, Pepin demonstrates the plausibility of this hypothesis. The evidence is bolstered by outbreaks of hepatitis that followed the mass-treatment campaigns. These efforts controlled target diseases, but they provoked a vigorous, silent amplification of HIV to the point that its sexual transmission could explode.

How did the virus cross the Atlantic? Again using social history, mathematics and molecular biology, Pepin conceives of a single "patient zero" among thousands of Haitians who helped in African education programs. Again, he identifies an iatrogenic factor that could have played a role in amplification: the practice of paying plasmapheresis donors for pooled blood products. Hematologists downplay the risk of infection from ordinary blood donation, but several well documented outbreaks of hepatitis $\mathrm{B}$ and other infections tracked commercial donation through pheresis. To cut costs, companies reused disposable apparatus or they returned pooled cells (and viruses) to donors. The argument is compelling.

The book closes with a chapter on "lessons learned." The greatest danger to humans is other humans, especially when they mess with nature. Pepin questions the wisdom of spending millions on space travel while pressing problems of disease and environment need greater monetary investment. He asks if travel beyond our dear planet will introduce another sinister pathogen. 
Pepin's message is clear. Even the best-intentioned efforts of the so-called developed world aggravated, and possibly provoked, the pandemic of AIDS. This book is essential reading for practitioners and students who care about global health.

But Pepin did not address another iatrogenic theory that would strengthen his argument. In his Histoire $d u$ sida (Payot; 1989), physician-historian Mirko Grmek articulated the notion of "pathocenosis," a stable mix of pathogens in time and place: when one vanishes, an unstable, ecological "hole" remains. From an opposite direction that brought him to the same place as Pepin, Grmek mused that successful immunization against diphtheria, measles, mumps, polio — and smallpox to which we could add the African campaigns that Pepin describes so well — created a "pathocenotic" window for HIV.

Grmek died in 2000 believing that molecular biology had generated a new paradigm for the conceptualization of disease and its treatment. Pepin's wonderful book shows that these scientific discoveries have also created powerful new tools for exploring the past. Read it.

\section{Jacalyn Duffin MD PhD}

Professor

Hannah Chair of the History of Medicine Queen's University

Kingston, Ont.

CMAJ 2012. DOI:10.1503/cmaj.120284 